\title{
VULNERABILIDADE SOCIAL E INDIVIDUAL E A GRAVIDEZ NA ADOLESCÊNCIA*
}

\section{SOCIAL AND INDIVIDUAL VULNERABILITY AND TEENAGE PREGNANCY}

\section{VULNERABILIDAD SOCIAL E INDIVIDUAL Y EMBARAZO ADOLESCENTE}

\author{
Márcia da Silva do Nascimento ${ }^{1}$, Umberto Gazi Lippi ${ }^{2}$, Álvaro da Silva Santos ${ }^{3}$
}

\begin{abstract}
RESUMO
Este estudo tem como objetivo traçar o perfil de vulnerabilidade individual e social e a susceptibilidade à gravidez na adolescência. Trata-se um estudo descritivo com amostra não probabilística realizado em cinco Unidades Básicas de Saúde do município de São Paulo em novembro de 2013. Foram sujeitos de pesquisa, 50 adolescentes grávidas. Dentre os principais resultados de vulnerabilidade individual tem-se: idade variou entre 14 a 19 anos, $28 \%$ referiu ter utilizado contraceptivos para evitar a atual gestação; na vulnerabilidade social tem-se: renda per capita de meio salário mínimo, $48 \%$ tinham menos de 8 anos de estudo, $64 \%$ não estudavam e, $60 \%$ não trabalhavam. São vários os determinantes para a gravidez na adolescência, o comportamento é apenas um. Há necessidade de intervenções voltadas a adolescente, sua família e comunidade no empoderamento destes; além da consciência do que interferem no comportamento pessoal e do grupo que estão inseridas.
\end{abstract}

Palavras-chave: Adolescente; Gravidez na adolescência; Vulnerabilidade em saúde; Vulnerabilidade social.

\footnotetext{
${ }^{1}$ Enfermeira mestre em Ciências da Saúde pelo IAMSPE, Especialista em Saúde Coletiva com ênfase em Saúde da Família; especialista em Formação de Docente para o Ensino Superior.

${ }^{2}$ Graduado em Medicina pela USP e doutorado em Medicina (Obstetrícia). Professor titular da Universidade Metropolitana de Santos, docente da Universidade Cidade de São Paulo e no IAMSPE.

${ }^{3}$ Graduado em Enfermagem, Especialização em Saúde Pública e Enfermagem Médico Cirúrgica, Pós-graduado em Psicanálise, Mestrado em Administração em Serviços de Saúde e Doutorado em Ciências Sociais. PósDoutorado em Serviço Social, na área de Idoso e Sociedade, estágio na Universidade de Barcelona-Espanha. Professor da UFTM no Curso de Mestrado e Doutorado em Atenção à Saúde, no Curso de Mestrado em Psicologia, e, no Curso de Graduação em Enfermagem.
}

\section{ABSTRACT}

* Este artigo foi extraído de um projeto maior desenvolvido em parceria com a Secretária Municipal da Saúde de São Paulo intitulado: Vulnerabilidade e gravidez na adolescência: análise espacial em uma região da periferia de São Paulo. 
This study aims to trace the profile of individual and social vulnerability and susceptibility to pregnancy in adolescence. This is a descriptive study with a non-probabilistic sample carried out in five Basic Health Units of the municipality of São Paulo in November 2013. Fifty pregnant adolescents were studied. Among the main results of individual vulnerability are: age ranged from 14 to 19 years, $28 \%$ reported using contraceptives to avoid the current gestation; In social vulnerability we have: per capita income of half a minimum wage, $48 \%$ had less than 8 years of study, 64\% did not study and 60\% did not work. There are several determinants for teenage pregnancy, behavior is just one. There is a need for interventions aimed at adolescents, their families and communities in their empowerment; Beyond the consciousness of what interferes in the personal and group behavior that are inserted.

Keywords: Adolescent; pregnancy in adolescence; Health vulnerability; Social vulnerability.

\section{RESUMEN}

Este estudio tiene como objetivo trazar el perfil de vulnerabilidad individual y social y la susceptibilidad al embarazo en la adolescencia. Se trata de un estudio descriptivo con muestra no probabilística realizado en cinco Unidades Básicas de Salud del municipio de São Paulo en noviembre de 2013. Fueron sujetos de investigación, 50 adolescentes embarazadas. Entre los principales resultados de vulnerabilidad individual se tiene: la edad varía entre 14 a 19 años, el 28\% refirió haber utilizado anticonceptivos para evitar la actual gestación; En la vulnerabilidad social se tiene: ingreso per cápita de medio salario mínimo, $48 \%$ tenían menos de 8 años de estudio, el $64 \%$ no estudia y el $60 \%$ no trabajaba. Son varios los determinantes para el embarazo en la adolescencia, el comportamiento es sólo uno. Hay necesidad de intervenciones dirigidas a adolescentes, su familia y comunidad en el empoderamiento de éstos; Además de la conciencia de lo que interfieren en el comportamiento personal y del grupo que están insertadas.

Descriptores: Adolescente; Embarazo en adolescencia; Vulnerabilidad en salud; Vulnerabilidad Social.

\section{INTRODUÇÃO}

Para a Organização Mundial da Saúde (OMS) a adolescência compreende o período entre 10 e 19 anos de idade. É caracterizado por diversas mudanças físicas (surgimento de caracteres sexuais secundários, finalização do crescimento e desenvolvimento morfofisiológico), além de transformações psicológicas, cognitivas e sociais. ${ }^{1}$

Embora, a maternidade na adolescência seja um fenômeno histórico. Em virtude da sua implicação social, nas últimas décadas tem-se intensificado o empenho pela redução da fecundidade na adolescência. ${ }^{1}$

Desde 1940, no Brasil, houve declínio da fecundidade com redução de um pouco mais de seis filhos para pouco mais de dois por mulher no início do século XXI. ${ }^{2}$ Contudo, manteve-se o índice de natalidade no grupo de adolescente entre 15 a 19 anos e houve pequeno acréscimo no de 10 a 14 anos de idade. Uma possível explicação para essa situação é a de que a atividade sexual está se iniciando cada vez mais cedo. ${ }^{1}$ 
$\mathrm{O}$ fato de engravidar mais precocemente chama a atenção, já que o papel da mulher na sociedade mudou. ${ }^{3}$

Atualmente, a inclusão feminina no mercado de trabalho pode ser vista como um evento socializador, o que por décadas ocorreu somente entre os homens. Esperase que a adolescente estude, trabalhe e não que engravide e tenha filhos. ${ }^{3}$

A gravidez na adolescência do ponto de vista biopsicossocial é determinada precoce, sendo um dos eventos mais preocupantes relacionadas à sexualidade nessa fase. Pode determinar agravos de ordem familiar e social, com graves efeitos para a vida da mãe adolescente, filho e família. ${ }^{3}$

Pressupõe-se que a gravidez na adolescência pode estar relacionada à lacuna de informação, orientação, instrução em sexualidade; às restrições de acesso aos serviços de saúde e aos insumos para a contracepção. Para muitas adolescentes, o advento da gravidez pode ser compreendido como a tentativa de encontrar e manter um espaço social, sobretudo em espaços marcados por desigualdades de gênero, raça e classe social. ${ }^{4}$ Além disso, a adolescência é uma faixa etária de alto risco para a transmissão e infecções sexualmente transmissíveis (IST). ${ }^{4}$
No Brasil dos anos 2000 até 2015, dados oficiais mostram que cerca de $20 \%$ dos nascidos vivos são filhos de mães adolescentes. ${ }^{5}$

O aumento da vulnerabilidade está relacionado a fatores que são comuns na adolescência. Autores referem à adolescência associada a condições de vulnerabilidade, como infecções sexualmente transmissíveis, o uso de álcool e droga, necessidade de descobrir o novo, baixa condição socioeconômica, iniciação sexual precoce, gravidez na adolescência e suas implicações indesejáveis. ${ }^{6,7}$

Nas pesquisas em saúde o termo vulnerabilidade, comumente é utilizado para indicar a antecedência dos riscos a agravos de saúde. O risco nos estudos tradicionais em epidemiologia tem identidade sólida, com caráter eminentemente analítico. ${ }^{7}$ Diferentemente do conceito de risco, o conceito de vulnerabilidade aprecia fatores não só individuais, mas ainda sociais e coletivos.

A vulnerabilidade pode ser analisada em suas três dimensões interdependentes: individual; programática; e social. Estas dimensões são apenas consideradas separadamente para fins analíticos e didáticos, porém na realidade são indissociáveis. A dimensão individual diz respeito à percepção 
individual de risco e o comportamento para a autoproteção. A dimensão programática se refere aos esforços e ações da própria organização visando a prevenção e a promoção da saúde. Por sua vez, a dimensão social diz respeito ao acesso à informação de forma mais vasta, gastos com serviços sociais e focaliza suas ações na redução das injustiças sociais. ${ }^{7}$

Considerando que as práticas sexuais na adolescência estão ocorrendo cada vez mais cedo e as condições nas quais os adolescentes estão expostos, o estudo buscou traçar o perfil de vulnerabilidade individual, social e a susceptibilidade à gravidez na adolescência.

\section{MÉTODO}

Trata-se de um estudo descritivo com amostra não probabilística constituída a partir de pesquisa de campo em Unidades Básicas de Saúde (UBS).

Para composição dos sujeitos da pesquisa, a Coordenadoria Regional de Saúde Leste (CRSL), que é o departamento responsável pelas Unidades de Saúde local indicou cinco UBS, considerando nestas unidades a alta incidência de gravidez na adolescência.

Foram sujeitos da amostra por conveniência 50 adolescentes grávidas, de qualquer idade gestacional cadastradas no programa de pré-natal das cinco UBS do bairro Cidade Tiradentes, localizadas no extremo leste do município de São Paulo. Foram excluídas adolescentes gestantes com déficit cognitivo.

O bairro Cidade Tiradentes apresenta alta concentração populacional (16.309,67 hab./Km2), com população estimada de 223.236 habitantes, sendo 41.274 (18,5\%) adolescentes, na qual $20.440(9,1 \%)$ adolescentes do sexo feminino, e dessas, 9.414 (4,2\%) estão na faixa etária de 10 a 14 anos e 11.026 $(4,9 \%)$ na faixa etária de 15 a 19 anos. $^{8}$

A região apresenta uma das maiores taxas de crescimento da cidade e graves problemas sociais. Esta população contabiliza um total de 60.740 famílias residentes no território abrangido pela respectiva subprefeitura e do total, 16.692 famílias encontram - se em situação de alta vulnerabilidade social e 2.598 famílias muito alta vulnerabilidade social. ${ }^{8,9}$

O desenvolvimento do estudo obedeceu aos preceitos éticos dispostos na resolução $n^{\circ} 466$, de 12 de dezembro de 2012, que se refere aos princípios norteadores de estudos envolvendo seres humanos. ${ }^{10}$

A pesquisa foi aprovada pelos Comitês de Ética em Pesquisa do IASMPE sob o parecer $n^{\circ} 457.635 / 2013$ e CAAE $n^{\circ}$ 21685213.9.0000.5463, e da SMS-SP sob 
o parecer $\mathrm{n}^{\circ} 459.866 / 2013$ e CAAE $\mathrm{n}^{\circ}$ 21685213.9.0000.5463.

A coleta de dados ocorreu em novembro de 2013. Foram selecionadas aleatoriamente 10 gestantes de cada uma das cinco UBS. Depois de esclarecidas sobre o objetivo da pesquisa e concordado pelas gestantes, as maiores de 18 anos assinaram o Termo de Consentimento Livre e Esclarecido (TCLE) e as menores de 18 anos assinaram o Termo de Assentimento e os seus responsáveis legais assinaram o Termo de Consentimento Livre e Esclarecido. $\mathrm{Na}$ sequência responderam individualmente ao instrumento para coleta de dados com questões fechadas, criadas pelos autores.

$\mathrm{O}$ roteiro de entrevista foi testado em julho de 2013 com dez adolescentes gestantes em UBS diferentes das contempladas na pesquisa, na qual não fizeram parte da análise deste estudo. Não foi necessário adaptações no roteiro de entrevista por ser avaliado como adequado no teste piloto. As variáveis buscaram traçar a suscetibilidade a gravidez na adolescência, segundo o perfil da vulnerabilidade individual e da vulnerabilidade social, apresentadas nas tabelas 1 e 2 , respectivamente.

Os resultados foram tabulados por meio de frequência absoluta e relativa no programa Excel, e analisados, segundo o método positivista que permite observar os fenômenos e fixar as ligações que possam existir entre eles ${ }^{11}$, com base na qual é possível entender características do fenômeno gravidez na adolescência.

\section{RESULTADOS}

Foi pesquisado um total de 50 gestantes com variações de 14 a 19 anos e média de 18 anos (DP $\pm 1,3$ anos). Não houve recusa à participação neste estudo.

No estado conjugal, prevaleceu a união consensual com 52\%, seguido de solteiras com $30 \%$ e separadas em $8 \%$. A idade média da coitarca das entrevistadas foi de 15,2 anos (DP $\pm 1,6$ anos); 76\% referem estar na primeira gravidez, $18 \%$ na segunda e $6 \%$ delas na terceira gravidez; apesar disto, 28\% apontam ter utilizado métodos contraceptivos para evitar a atual gestação. Os pais eram separados em $60 \%$ das gestantes estudadas, $52 \%$ proferiu ter bom relacionamento com a família, $60 \%$ delas indicaram a mãe como orientadora sexual e $46 \%$ delas mencionam receber principal apoio financeiro e suporte emocional da mãe.

Observou-se a média de 4,4 pessoas por domicílio ( $\mathrm{DP} \pm 1,8$ ), renda familiar das gestantes de 2 a 3 salários mínimos, com média de 2,8 salários mínimos $(\mathrm{DP} \pm 0,9)$ e a renda média por pessoa de cada grupo familiar de gestante foi de $1 / 2$ salário 
mínimo. Quanto à escolaridade, não havia nenhuma analfabeta. No entanto, $48 \%$ das adolescentes tinham menos de oito anos de estudo, ou seja, não haviam completado o ensino fundamental. Eram $68 \%$ as que relataram morar em residência própria da família de origem e, $60 \%$ não trabalhavam; $64 \%$ não estudavam e $54 \%$ utilizavam a internet como meio de informação para a prática sexual.

Tabela 1: Caracterização dos Fatores da Vulnerabilidade Individual. São Paulo, 2013.

\begin{tabular}{lcc}
\hline Idade (anos) & $\mathbf{N}$ & $\mathbf{\%}$ \\
\hline 14 & 4 & 8 \\
15 & 1 & 2 \\
16 & 5 & 10 \\
17 & 5 & 10 \\
18 & 13 & 26 \\
19 & 22 & 44 \\
\hline
\end{tabular}

\begin{tabular}{lcc}
\hline Estado conjugal & & 62 \\
Viviam com companheiro & 31 & 38 \\
Não viviam com companheiro & 19 & 28 \\
\hline Idade da coitarca & 14 & 42 \\
De 11 a 13 anos & 21 & 30 \\
De 14 a 16 anos & 15 & 76 \\
De 17 a 19 anos & 38 & 18 \\
\hline Número de gestações & 9 & 6 \\
Uma & 3 &
\end{tabular}

Método contraceptivo utilizado na

tentativa de evitar gravidez atual

Anticoncepcional oral $\quad 8 \quad 16$

"Pílula do dia seguinte" $\quad 3 \quad 6$

$\begin{array}{lll}\text { Preservativo masculino } & 3 & 6\end{array}$

$\begin{array}{lll}\text { Nenhum } & 36 & 72\end{array}$

\begin{tabular}{lcc}
\hline Estado conjugal dos pais das gestantes & 36 & 22 \\
Casados & 11 & 14 \\
União consensual & 7 & 4 \\
Solteiros & 2 & 60 \\
Separados & 30 & 34 \\
\hline Grau de relacionamento com a família & 17 & 52 \\
Ótimo & 26 & 10 \\
Bom & 5 & 4 \\
Regular & 2 & 60 \\
Péssimo & & 12 \\
\hline Fonte de orientação acerca da sexualidade & 30 & 10 \\
Mãe & 6 & 4 \\
Familiares & 5 & 4 \\
Amigos & 2 & 8 \\
Professores & 2 & 2 \\
Profissionais da saúde & 4 & \\
Nunca obteve informações & 1 & 46 \\
Outros & & 6 \\
\hline Principal pessoa que oferece apoio & & \\
financeiro & 23 & \\
Mãe & 3 & \\
Pai & & \\
\hline
\end{tabular}

Rev Enferm Atenção Saúde [Online]. Jan/Jul 2018; 7(1):15-29 ISSN 2317-1154 
Familiares

6

12

Pai do bebê ou família paterna

36

Principal pessoa que oferece suporte

emocional

Mãe

Pai

Familiares

Profissionais de saúde

Amigos

Pai do bebê ou da família paterna

Nenhum

18

Total

\begin{tabular}{cc}
23 & 46 \\
2 & 4 \\
9 & 18 \\
2 & 4 \\
1 & 2 \\
11 & 22 \\
2 & 4 \\
\hline $\mathbf{5 0}$ & $\mathbf{1 0 0}$
\end{tabular}

Tabela 2: Caracterização dos Fatores da vulnerabilidade Social. São Paulo, 2013.

\begin{tabular}{|c|c|c|}
\hline $\mathrm{N}^{0}$ de pessoas por residência/gestante & $\mathbf{N}$ & $\%$ \\
\hline Até 2 pessoas & 17 & 34 \\
\hline De 3 a 4 pessoas & 15 & 30 \\
\hline De 5 a 6 pessoas & 13 & 26 \\
\hline De 7 a 9 pessoas & 5 & 10 \\
\hline \multicolumn{3}{|l|}{ Renda familiar/gestante* } \\
\hline Até1 Salário mínimo & 10 & 20 \\
\hline De 2 a 3 Salários mínimos & 37 & 74 \\
\hline De 4 a 5 Salários mínimos & 2 & 4 \\
\hline Acima de 5 Salários & 1 & 2 \\
\hline \multicolumn{3}{|l|}{ Nível educacional } \\
\hline Fundamental incompleto & 24 & 48 \\
\hline Fundamental completo & 2 & 4 \\
\hline Médio incompleto & 13 & 26 \\
\hline Médio completo & 10 & 20 \\
\hline Superior incompleto & 1 & 2 \\
\hline \multicolumn{3}{|l|}{ Tipo de residência das gestantes } \\
\hline Alugada & 16 & 32 \\
\hline Própria da família de origem & 34 & 68 \\
\hline \multicolumn{3}{|l|}{ Trabalha durante a gravidez } \\
\hline Sim & 20 & 40 \\
\hline Não & 30 & 60 \\
\hline \multicolumn{3}{|l|}{ Estuda durante a gravidez } \\
\hline Sim & 18 & 36 \\
\hline Não & 32 & 64 \\
\hline \multicolumn{3}{|c|}{$\begin{array}{l}\text { Meio de comunicação para informação da } \\
\text { prática sexual }\end{array}$} \\
\hline Internet & 27 & 54 \\
\hline Televisão & 11 & 22 \\
\hline Nenhum & 6 & 12 \\
\hline Outros & 5 & 10 \\
\hline Livros didáticos & 1 & 2 \\
\hline Total & 50 & 100 \\
\hline
\end{tabular}

*Renda per capita de 1/2 salário mínimo: valor de referência $\mathbf{R} \$ \mathbf{6 7 8 , 0 0}$

\section{DISCUSSÃO}

No município de São Paulo a faixa etária de 10 a 14 anos é a que mais cresce Rev Enferm Atenção Saúde [Online]. Jan/Jul 2018; 7(1):15-29 na frequência de gravidez, apresentando acréscimo de 9\% entre os anos de 2004 e 2013. ${ }^{12}$ Enquanto observa-se um acréscimo 
nas faixas etárias extremas, nota-se um decréscimo de aproximadamente 4,5\%, na taxa de fecundidade na faixa etária entre 20 e 34 anos. $^{12}$

Segundo o Boletim da Coordenação de Epidemiologia e Informação (CEInfo), na região onde foi realizada a pesquisa, foram registrados 3806 nascimentos em 2013, sendo 753 (19,8\%) nascidos de mães adolescentes e desse total, $31(4,1 \%)$ partos em meninas de 10 a 14 anos. ${ }^{12}$ Embora esse estudo não seja probabilístico no grupo de pesquisa foi encontrado $8 \%$ de gestantes nesta faixa etária, muito acima dos dados apresentados no boletim CEInfo.

Os informes sobre o atendimento do SUS demonstram também o alto número de internações para atendimento obstétrico na faixa etária de 10 a 19 anos. Em 2013 as internações por gravidez, parto e puerpério corresponderam a $37 \%$ das internações entre mulheres nessa faixa de idade. $^{4}$

É comum a ocorrência de nascidos vivos em situação de risco relacionado à idade das mães, tais como prematuridade e baixo peso ao nascer. Em 2013, no município de São Paulo, a percentagem de baixo peso ao nascer $(<2500$ g) dos nascidos de mães de 10 a 14 anos foi $14,3 \%$ e no de 15 a 19 anos foi de $10,8 \%$, evidenciando maior prevalência de recémnascido ( $\mathrm{RN}$ ) de baixo peso e maiores intercorrências perinatais entre adolescentes mais jovens, comparadas às adolescentes com mais idade. ${ }^{12}$

O casamento ou a união consensual é uma situação frequentemente vivenciada pelas adolescentes entrevistadas. Embora a pouca idade das gestantes, a maioria (62\%) delas mencionou morar com o pai do seu filho e $8 \%$ ter coabitado com um parceiro e já ter se separado.

A experiência da união induz as adolescentes a abrirem mão de suas expectativas. Apesar de atualmente, ser percebida de forma diferente, a virgindade, o casamento, a maternidade, o amor, os papéis sexuais nas afinidades conjugais e sociais, ainda existe por parte de alguns grupos sociais, uma influência para que o casal formalize a união e venha a conviver na mesma residência, ainda que seja sem ter independência financeira. ${ }^{13}$

Em um estudo de gravidez na adolescência, considerando as diferenças socioeconômicas foi observado que enquanto a maior parte das adolescentes da classe A continuava solteira, na classe D este comportamento era oposto, visto que a maioria passava a morar com seu companheiro. ${ }^{13}$ Esse fator traduz o modo como as pessoas nas diferentes classes socioeconômicas entendem a convivência afetiva originadas pela gravidez precoce. 
O grupo de estudo mostra nítida iniciação sexual precoce. A Pesquisa Nacional sobre Saúde dos Escolares $(\mathrm{PENSE})^{14}$, observou que, entre adolescentes de 13 a 15 anos, a iniciação sexual ocorreu mais cedo naqueles que residiam sem os pais ou em lares uniparentais, comparados aos que moravam com pai e mãe. A coitarca precoce deixaria os adolescentes ainda mais vulneráveis para comportamentos de risco, por ser um período crítico de transição entre a infância e a idade adulta. Nesta ocasião, a cognição e a capacidade de decisão ainda estão em desenvolvimento. ${ }^{1}$

Observou-se, porém, que $76 \%$ delas realmente estavam na primeira gestação, mas, surpreendentemente, $18 \%$ encontravam-se na segunda e 6\% na terceira gestação. Por serem as mulheres objeto desta pesquisa muito jovens, seria de se esperar que fossem primíparas em sua quase totalidade e com poucos casos de reincidência.

A repetição da gravidez na adolescência é frequente no Brasil e no mundo, conforme outras pesquisas, sendo que a baixa escolaridade materna, a troca de parceiros e as uniões incertas foram ocorrências acentuadas para a reincidência da gestação. ${ }^{15,16,17}$
A primeira gravidez não é um fato expressivamente suficiente para afastar a ocorrência de novas gestações. Uma vez que alguém assumiu os cuidados da prole anterior, a jovem avalia como apropriada a receptividade do bebê pela família, deixando-a em uma condição aparentemente cômoda e segura para uma nova gravidez. Contam, outra vez, com pessoas próximas, nos cuidados com o outro filho. ${ }^{17}$

Um número expressivo de gestantes referiu não ter utilizado qualquer método contraceptivo para evitar a atual gravidez. Embora a adolescente tenha informação e muitas vezes possuir nível de escolaridade e do conhecimento plausível sobre sexualidade, as adolescentes não conseguem converte-los em sexo seguro e em mudança de prática. ${ }^{16}$

Além das questões de vulnerabilidade social, a gravidez pode derivar da condição de vulnerabilidade individual, como do uso inadequado ou falta de conhecimento do método contraceptivo, desconhecimento da fisiologia da reprodução e dos efeitos das relações sexuais, por não acreditar nos riscos da gravidez precoce ou redução da capacidade de julgamento por razão do efeito de bebidas alcoólicas e drogas, entre outros. $^{16}$ 
Dessa forma é possível conferir que, exclusivamente, a informação sobre os riscos da gravidez precoce não é satisfatória para que os jovens desenvolvam um comportamento sexual seguro. É necessário ir mais adiante da simples informação reprodutiva ou anticonceptiva. $^{17}$

Das jovens entrevistadas foi observado que $64 \%$ dos pais das gestantes não conviviam com as mesmas, $60 \%$ devido estarem separados e $4 \%$ por serem solteiros, $22 \%$ estavam casados e $14 \%$ em união consensual.

A ausência da convivência dos pais pode representar uma lacuna na vida da jovem, na qual idealiza a maternidade como opção de obter uma família na sua totalidade. $^{18}$

Quanto ao relacionamento familiar, houve predomínio das respostas de ótimo e de bom relacionamento. A consistência dessa relação familiar não parece ter sido suficiente para evitar uma gravidez precoce. A gravidez na adolescência é estimulada por situações internas e externas, sendo um dos principais, o tipo de relacionamento familiar. Na maioria das vezes as informações dadas pela família não estão pautadas em achados científicos e sim em modelos familiares. ${ }^{18}$

A mãe foi percebida como principal fonte de apoio financeiro e emocional, bem como a que trazia informações sobre sexualidade. É vasta a produção científica acerca dos temas sexualidade, saúde reprodutiva e sexual, contudo poucos trabalhos referem-se ao conhecimento e prática dos docentes que trabalham com orientação sexual para adolescentes. Nas escolas públicas um estudo encontrou-se deficiência em sua formação, embora haja disponibilidade de informações, por meio de publicações científicas e oficiais. ${ }^{19}$

De acordo com o Índice Paulista de Vulnerabilidade Social (IPVS), indicador que classifica a vulnerabilidade, as famílias de baixa renda são aquelas com renda igual ou inferior a $1 / 2$ salário mínimo per capita ou renda familiar mensal de até três salários mínimos, assim podemos verificar que as gestantes compreendidas nesse estudo são classificadas como de baixa renda, em condição de vulnerabilidade social com risco a saúde materno-infantil. ${ }^{9}$ Contudo engravidam tanto as adolescentes de classe social mais baixa, quanto as de classe mais alta, diferenciando apenas na maneira de lidar com a circunstância. Infelizmente são escassos os trabalhos acerca das adolescentes de classe socioeconômica mais elevada já que raramente ocorre coleta de dados para estudos, nos serviços privados que comumente frequentam. 
31,8\% das residências da região de pesquisa estão localizados em área de alta e muito alta vulnerabilidade (IPVS 5-6), 25,9\% em situação de baixa renda, 6,5\% em situação de pobreza e 1,4\% em extrema pobreza $^{9}$, determinantes sociais que poderão expor as adolescentes e seus filhos a maiores riscos de doença e morte.

Embora a maior parte das pesquisadas estivesse entre 18 e 19 anos, idade já compatível para o início do ensino superior, somente uma referiu ter iniciado a faculdade. Observou-se no grupo pesquisado baixa escolaridade, que é um fator que influencia alterações no ciclo gravídico puerperal. Esses dados corroboram com os dados do Sistema de Informação sobre Nascidos Vivos (SINASC), o qual mostra entre os períodos de 2000 a 2009, que as mulheres brasileiras com menor escolaridade apresentaram menor proporção de nascidos vivos, menos de sete consultas de prénatal, representando a maior proporção sem acompanhamento na gestação. ${ }^{5}$

Estudos revelam que a baixa escolarização tem relação com a baixa condição socioeconômica das gestantes, a precariedade do nível educacional pode perpetuar a tendência à pobreza, com acentuação dos riscos sociais aos quais mães adolescentes e seus filhos estão submetidos, como também interferem na percepção das mães sobre a importância da assistência a saúde materno-infantil. ${ }^{13}$

A evasão escolar agregada à precocidade da gravidez traz sérias implicações para a adolescente, para seu filho e para a sociedade em geral, sobretudo já que, nessa faixa etária, uma das raras alternativas de inclusão social e de promoção econômica se dá por meio do sistema educacional. Caso contrário, entram no mercado de trabalho sem formação e por isso são pouco remuneradas. $^{12}$

A adolescente que engravida e não recebe apoio familiar nem da sociedade tem grande possibilidade de abandonar os estudos. ${ }^{13}$ Contudo, isso não significa que a família proponha-se sempre a assumir os cuidados e encargos de seu filho.

A maternidade na adolescência é idealizada como uma opção para o sucesso pessoal e familiar e cria a fantasia de que não há mais necessidade dos estudos, o que dificulta o retorno à escola. ${ }^{14}$

Quanto à caracterização do domicílio, mais da metade das entrevistadas declararam morar em residência própria da família de origem e $32 \%$ disseram morar em residência alugada. Muitas vezes os pais adolescentes permanecem habitando com a família de origem, devido às dificuldades financeiras, uma vez que, a maioria deles não tem 
estabilidade profissional, em decorrência da idade e do contexto social no qual estão inseridos, tornando-se dependentes do apoio da família. ${ }^{13}$

Nas classes menos favorecidas, mãe, criança e frequentemente também o pai do bebê passam a morar com as famílias de origem, apresentando-se, múltiplos centros familiares coabitando num mesmo recinto, compondo e decompondo a renda e a disposição familiar. ${ }^{13}$

Segundo a Fundação Sistema Estadual de Análise de Dados (Fundação SEADE), $14,3 \%$ dos domicílios de São Paulo estão localizados em áreas classificadas como de alta e muito alta vulnerabilidade (grupos 5 e 6 do IPVS ). ${ }^{9}$

O grupo 6 do IPVS concentra apenas áreas com aglomerados subnormais com concentração de população jovem e de baixa renda. A maior parte deles se encontra nas regiões sul e leste do município de São Paulo. ${ }^{9}$ Além de não estudarem, a maioria das pesquisadas também não trabalhavam. No geral, observa-se que, independentemente da faixa de idade, provavelmente a evasão escolar está relacionada à precocidade da gestação, resultados também encontrados em estudos, nos quais relatam que poucas adolescentes regressam aos estudos depois da gravidez, originando níveis educacionais menores e, portanto condição inadequada de profissionalização, tendência a famílias numerosas e outras tantas modificações na vida. ${ }^{13}$

A gestação precoce pode trazer desvantagens à trajetória educacional da gestante, contribuindo para a evasão escolar e dificultando o retorno à escola, limitando o seu progresso acadêmico e as possibilidades de adequação ao mercado de trabalho. ${ }^{13}$ Sem fonte de renda a gestante dependerá de outra pessoa para o seu sustento, favorecendo uma condição de vulnerabilidade.

O meio de comunicação mais utilizado pelas adolescentes para obter conhecimento acerca da sexualidade foi a internet seguido da televisão. Compreendese que os meios de comunicação social possuem poderosa influência, não apenas sobre a população adulta, mas, também sobre os jovens, exercem fortes influências sobre a percepção do comportamento e a realidade social. Além de atrair muito os jovens o assunto sobre sexualidade, induz ao aceleramento do acesso ao mundo adulto, ainda que o adolescente não tenha entendimento cognitivo, capacidade emocional e psíquica para tal. ${ }^{20}$

\section{CONCLUSÃO}

Através dessa pesquisa conclui-se que a maioria das adolescentes grávidas do grupo de estudo são mulheres de 15 a 19 
anos, coabitam com o parceiro, residem com a família de origem e estão na primeira gestação. Não trabalham e nem estudam, têm internet como principal meio de comunicação e a mãe é a principal fonte de orientação sexual, de apoio financeiro e emocional.

Foi possível identificar que as vulnerabilidades determinantes para a gravidez na adolescência é um conjunto de condições, dentre as quais o comportamento é apenas uma. Não há como falar em intervenções voltadas apenas para a adolescente, sem ter consciência das situações que interferem em seus comportamentos pessoais e do grupo da qual estão inseridas, sem acessar os elementos externos que podem apoiar e direcionar as pessoas numa perspectiva de maior ou menor autoproteção.

Neste estudo é notória a necessidade de intervenções voltadas ao empoderamento da adolescente, da família e da comunidade. Contudo o mesmo apresentou limitações importantes em relação ao tamanho da amostra, que ao se apresentar em número diminuído permite considerar os resultados encontrados, exclusivamente para a população em questão. Outra limitação importante é a precisão conceitual de vulnerabilidade, fator crucial para uma análise séria e explicativa da realidade. Todavia, há em diversos trabalhos nenhuma ou insuficiente discussão acerca de seu significado.

Por fim, o tema estudado tem grande relevância social e compreende os possíveis aspectos das vulnerabilidades para a gravidez na adolescência, contribuindo para maiores intervenções de políticas locais.

\section{REFERÊNCIAS}

1. World Health Organization - WHO. (2015, Março 22). Adolescent pregnancy. [Citado em 24 out 2016].

Disponível em: http://www.who.int/ mediacentre/factsheets/fs364/en/.2. Censo, 2. Índice Brasileiro de Geografia e Estatísticas (IBGE). Síntese de indicadores sociais: uma análise das condições de vida da população brasileira. Rio de Janeiro; 2010. [Citado 25 de maio 2017] Disponível em: http://www.censo2010. ibge. gov. br 3. Organização Mundial da Saúde (Brasil). Nossas prioridades: Adolescentes [internet]. Brasília (DF): UNICEF; 2011. [Citado em 24out2013]. Disponível em: http://www.unicef.org/brazil/pt/activities_9 994.htm

4. Ministério da Saúde (Brasil). Secretaria de atenção à Saúde. O SUS e a saúde sexual e reprodutiva de adolescentes e jovens no Brasil. Brasília out. 2013. 5. Ministério da Saúde (Brasil). Secretaria de Vigilância em Saúde - Departamento de Análise de Situação de Saúde - - Sistema de Informações sobre Nascidos Vivos (SINASC). [Citado em 20 jan 2017] Disponível em:

http://www.saude.sc.gov.br/cgi/tabcgi.exe? sinasc.def

6. Ayres JRM, Carvalho YM,

Nasser MA et al. Caminhos da integralidade: adolescentes e jovens na Atenção Primária à Saúde. Interface comum saúde educ. v. 16, n. 40, 2012. p. 
67- ${ }^{8} 1$. [Citado em 25 maio 2017].

Disponível em:

http://www.producao.usp.br/handle/BDPI/ 39737

7. Bertolozzi MR, Nichiata LYI, Ciosak

SI, Hino P, Val LF et al. Os conceitos de

vulnerabilidade e adesão na Saúde

Coletiva. Rev esc enferm da USP

[Internet]. 2009; [Citado 25 de maio 2017]

43(2):1326-30. Disponível em:

http://www.scielo.br/pdf/reeusp/v43nspe2/

a31v43s2.pdf

8 Coordenadoria do Observatório de

Políticas Sociais (Smads) [Internet]. In:

Assistência e Desenvolvimento Social.

Atlas Socioassistencial da cidade de São

Paulo: SP. 2015. Disponível em:

http://www.prefeitura.sp.gov.br/cidade/sec

retarias/upload/assistencia_social/arquivos/

atlas_socioassistencial_sp_2015.pdf

9. Coordenação da Subprefeitura do

Município de São Paulo. Subprefeitura

Cidade Tiradentes. 2016 [Internet]. São

Paulo: SP. [Citado 25 maio 2017].

Disponível em:

http://www.prefeitura.sp.gov.br/cidade/sec

retarias/subprefeituras/cidade tiradentes/hi

storico/index.php? $\mathrm{p}=94$

10. Conselho Nacional de Saúde (Brasil).

Resolução n. 466, de 12 de dezembro de

2012. [internet]. Brasília (DF): CNS; 2012.

[Citado em 18 jan. 2014]. Disponível em:

http://conselho.saude.gov.br/resolucoes/20

12/Reso466.pdf

11. Turato ER. Métodos qualitativos e

quantitativos na área da saúde: definições,

diferenças e seus objetos de pesquisa. Rev

Saúde Pública 2012; 39 (3): 507-14.

12. Boletim da Coordenação de

Epidemiologia e Informação [Internet]. In:

Secretaria Municipal da Saúde (São

Paulo): 2014, [Citado em 9 maio 2015].

Disponível em:

http://www.prefeitura.sp.gov.br/cidade/sec retarias/upload/saude/arquivos/publicacoes

/Boletim_CEInfo_Dados_2014.pdf

13. Taborda JÁ, Silva FC; Ulbricht L,

Neves EB. Consequências da gravidez na

adolescência para as meninas

considerando-se as diferenças socioeconômicas entre elas. Cad. Saúde

Colet. Rio de Janeiro [internet]. 2014

[Citado 9 maio 2015]; 22 (1): 16-24.

Disponível em:

http://www.scielo.br/pdf/cadsc/v22n1/1414

-462X-cadsc-22-01-00016.pdf

14. Sasaki RSA, Souza MM, Leles CR,

Malta DC, Sardinha LMV, Freire MCM.

Comportamento sexual de adolescentes

escolares da cidade de Goiânia, Goiás.

Rev. bras. Epidemiol. [internet].

2014;[Citado em 10 dez. 2015]; 17 Esp 1:

172-182. Disponível em:

http://dx.doi.org/10.1590/1809-

4503201400050014

15. Silva AAA, Coutinho IC, Katz L,

Souza ASR. Fatores associados à

recorrência da gravidez na adolescência em uma maternidade escola: estudo casocontrole. Cadernos de Saúde Pública, Rio de Janeiro [internet].2013[Citado em 16 dez 2014]; 29(3): 496-506. Disponível em: http://www.scielo.br/scielo.php?script=sci arttext\&pid=S010211X2013000300008\& lng=en\&nrm=iso

16. Moura LNB, Gomes KRO, Sousa

CRO, Maranhão TA. Multiparidade entre adolescentes e jovens e fatores de risco em Teresina/Piauí. Adolesc Saude.

2014[Internet].;11(3):51-62. [Citado 9 de mar 2016];24(3):320-26. Disponível em:

http://www.adolescenciaesaude.com/detalh e artigo.asp?id=456\#

17. Velho MTC, Riesgo I, Zanardo CP, Freitas AP, Fonseca R. A reincidência da gestação na adolescência: estudo retrospectivo e prospectivo em região do sul do Brasil. Revista Saúde e Pesquisa, [internet]. 2014, [Citado 22 janeiro 2015]; 7 (2), p. 261-273. Disponível em: http://periodicos.unicesumar.edu.br/index. php/saudpesq/article/viewFile/3509/2376 18 Sganzerla IM, Levandowski DC.

Ausência paterna e suas repercussões para o adolescente: análise da literatura.

Psicologia em Revista, [internet]. 2010.

[Citado em 20 de mar 2014]; 16(2), p. 295309. Disponível em:

http://pepsic.bvsalud.org/pdf/per/v16n2/v1 $\underline{6 \mathrm{n} 2 \mathrm{a} 05 . \mathrm{pdf}}$ 
19. Borges JPA, Ferreira MCM.

Orientação sexual para adolescentes:

conhecimento e Prática de docentes das

escolas públicas. Rev. Enferm Atenção

Saúde [Internet]. Jan/jun 2015. [Citado 27

de julho 2016]; 4(1):89-96. Disponível em:

http://seer.uftm.edu.br/revistaeletronica/ind

ex.php/enfer/article/download/1266/1137

20. Strasburger VC, Wilson BJ, Jordan

AB. Crianças, adolescentes e a mídia.

Tradução de Sandra Mallmann. Revisão

técnica: Márcia Benetti. 2. ed. Porto

Alegre: Penso [internet]. 2011. [Citado 13

de set 2015]. Disponível em:

http://www.grupoa.com.br/livros/comunica

cao/criancas-adolescentes-e-a-

midia/9788563899101

RECEBIDO: 22/12/2016

APROVADO: 29/01/2018

PUBLICADO: 07/2018 